\title{
ANÁLISIS DE LAS PRÁCTICAS EVALUATIVAS DESDE LA PERSPECTIVA DE LOS PROPIOS ACTORES Y ACTORAS EN LA REGIÓN ATLÁNTICA
}

\author{
Dra. Leda Badilla Chavarría
}

\section{Resumen}

Esta investigación evaluativa se propuso valorar, desde una perspectiva cualitativa, el componente "Evaluación Escolar" del Proyecto "Mejoramiento de la calidad de la Educación Ministerio de Educación / Banco Mundial" en la Zona Atlántica del país.

Participan diferentes actores y actoras de la comunidad educativa: equipos regionales docentes, padres, madres, alumnos y alumnas. Se utilizan diversas técnicas para la producción de la información. El análisis parte de las lógicas vivenciales en el proceso evaluativo que experimentan diferentes actores y actoras.

Por las características del estudio, se incluyen las voces de los y las participantes, y los textos se presentan tal y como fueron expresados por ellos.

\section{Abstract}

The purpose of the investigation was to evaluate from a qualitive perspective the Scholastic Evaluation component in the project conducted in the Atlantic area of the country involved different participants of the educational community: regional teams, advisors, educators, parents, students. Diverse techniques were used to complete the information. The analysis departs from the logical experiences of the actors in the evaluative process. Problems and solutions were also identified by the participants.

Because of the characteristics of the study the voices of the participants are included. The texts are presented the way they were expressed by the actors.

Descriptores: evaluación cualitativa, educación formal, evaluación sumativa, e investigación.

\footnotetext{
${ }^{1}$ Leda Badilla Chavaría. Doctora en Educación. énfasis en Evaluación. Universidad de Costa Rica. Directora del Programa de Postgrado en Evaluación Educativa desde 1994 hasta la fecha. Miembra titular de la Comisión Técnica de Evaluación, Sistema Carreras Regionales CSUCA desde 1998. Ha desarrollado diversas investigaciones evaluativas a nivel nacional. Labora en la Universidad de Costa Rica.
} 


\section{Introducción}

\section{L}

a zona Atlántica del país se caracteriza, entre otros aspectos, porque en ella habita el 74\% de la población de ascendencia afroantillana (Putman, 2004). La misma autora señala que la tercera parte de las cónyuges de los hombres afrocostarricenses, así como la cuarta parte de los cónyuges de las mujeres afrocostarricenses no son afrocostarricenses. Estas uniones mixtas tienen un significado especial en lo que a educación se refiere, especialmente en la socialización inicial y las consecuentes prácticas socioculturales, entre ellas, el uso del lenguaje. Asimismo, en la zona convergen grupos de origen oriental y población indígena entre otros.

Para algunos afrocostarricenses (Sawyers, Joycelyn. Comunicación personal, 2005), la inversión estatal en educación hacia la región Atlántica, podría ser mayor y más acelerada. Hay circulación migratoria hacia la zona y a esta dinámica no escapa el nombramiento temporal de educadores y educadoras y las conocidas implicaciones que esta situación genera en el proceso educativo.

En la década de los ochenta, se determina en Costa Rica un faltante de educadores graduados, situación que no sólo era preocupante en ese momento, sino que se proyectaba en cifras alarmantes para la década de los noventa. En 1993, el faltante de educadores de I y II Ciclos llega al 20 por ciento (MEP, 1994).

En ese entonces, el Gobierno de Costa Rica presenta, ante el Banco Mundial, un proyecto cuya meta final es el mejoramiento de la calidad de la educación. Con este fin, se crea una estructura técnico-organizativa que coordinara las acciones respectivas, conocida como el Programa de Mejoramiento de la Calidad de la Educación (PROMECE) ${ }^{2}$.

PROMECE inicia en 1993, con un proyecto que considera diferentes componentes, que conducirían al mejoramiento de la calidad del sistema educativo costarricense. Este programa tuvo cobertura nacional. La intención fue favorecer el área rural y las áreas urbano-rurales, de atención prioritaria, que estaban en franco desequilibrio frente al área urbana.

Un componente de especial interés en ese Proyecto lo constituyó la evaluación escolar. En la "Política Educativa hacia el Siglo XXI" vigente a la fecha, se hizo un replanteamiento-de dicho componente, pues la práctica evidenciaba una

\footnotetext{
2 Las primeras acciones se llevaron a cabo en el gobiemo Arias Sánchez (86-90). se concretaron y aprobaron en el gobierno Calderón Fournier (90-94) y se desarrollaron en el período Figueres Olsen (94-98). El gobierno Rodríguez Echeverría (98-2002), evaluó lo actuado, con el propósito de identificar los factores de éxito o fracaso. pero sobre todo determinar las lecciones aprendidas durante el desarrollo del programa en su componente "Evaluación Escolar". Los resultados de esta evaluación permiten generar insumos para futuros proyectos y retroalimentar a los diferentes actores sociales sobre los logros obtenidos.
} 
disociación entre los fundamentos filosóficos y los teóricos de la evaluación escolar. Esto se reflejó en la falta de coherencia entre los planes y programas vigentes, el proceso de evaluación, los recursos didácticos y materiales educativos al servicio de educadores y educadoras, estudiantes, de la formación, la capacitación y la mediación docente (lo didáctico y lo pedagógico).

Por lo tanto, se propuso implementar un sistema evaluativo que suministrara los indicadores del desarrollo de la calidad de la Educación General Básica. De esta forma, se logró capacitar a 29.102 funcionarios y funcionarias, entre docentes, directores y supervisores escolares, de ellos 4.800 eran miembros de los Comités de Evaluación. Se elaboraron, aplicaron y analizaron 361.000 pruebas diagnósticas y de acreditación para I, III, VI y IX grados, así como las pruebas de bachillerato (MEP, s.f.).

También se efectuó la capacitación a asesores regionales, maestros, comités de evaluación, estudiantes y padres de familia. Los resultados fueron difundidos por medio de las Direcciones Regionales.

En este componente se establecieron dos niveles de actividades. En el primer nivel, la labor se concentró en la capacitación del equipo central, actividad llevada a cabo con la asesoría del Instituto de Investigaciones para el Mejoramiento de la Educación Costarricense (IIMEC-UCR) y la especialización del recurso humano en el campo de la evaluación por medio de un Posgrado en Evaluación Educativa en la Universidad de Costa Rica, que logró graduar, en tres años, a 42 funcionarios con el grado de Maestría.

Lo planteado en este artículo, en la justificación y los presupuestos teóricos, obedece a un marco de referencia explícito en los documentos oficiales del Ministerio de Educación Pública. Deseo dejar constancia de que no necesariamente es el marco de referencia propio. Además, la evaluación se realiza diez años después.

\section{Algunos referentes administrativos por considerar}

El antecedente del "Programa de Pruebas" se ubica en la Administración Arias Sánchez (1986-1990), cuando la Universidad de Costa Rica y el Ministerio de Educación Pública firman un convenio para llevar a cabo pruebas diagnósticas en el sistema educativo costarricense. El convenio se sustenta en un proyecto de investigación. Con este Programa, se sientan las bases académicoorganizativas que sirvieron de referencia para el Programa de Pruebas. Se inicia así, un proceso de elaboración de pruebas diagnósticas en I, II y III ciclo, y se restaura el bachillerato en educación secundaria.

En la administración Calderón Fournier (1990-1994), se gesta y se firma el Convenio para el Mejoramiento de la Calidad de la Educación Costarricense 
(PROMECE), siendo uno de sus componentes la Evaluación Escolar. El énfasis se centra en consolidar un Programa de Pruebas Nacionales, que diagnosticara el estado de la educación en el primero, segundo y tercer ciclo. Como recomendación de la Comisión Decisoria, conformada por representantes de las universidades de Costa Rica, Nacional y Estatal a Distancia, el MEP y el CONARE se abre el Programa de Posgrado en Evaluación Educativa en la Universidad de Costa Rica.

En la administración Figueres Olsen (1994-1998), el Programa de Pruebas Nacionales alcanza su mayor desarrollo y se incorporan los graduados de la Maestría en Evaluación Educativa al proceso. La perspectiva de la evaluación se abre a otras áreas: destrezas cognoscitivas, pruebas para el primer ingreso y pruebas de salud física, entre otras. Se crea el Centro Nacional de Evaluación para la Educación (CENE-EDU), adscrito al MEP. Con esta decisión, se traslada el Programa de Pruebas de la Universidad de Costa Rica al Ministerio de Educación Pública.

En la administración Rodríguez Echeverría (1998-2002), se cierra el CENE-EDU y el programa de Pruebas Nacionales pasa a la División de Control de Calidad, del Ministerio de Educación.

\section{Elementos de carácter evaluativo que emanan de la Política Educati- va hacia el Siglo XXI}

Los documentos analizados destacan (MEP, 1994a; 1994b), entre los retos de la educación, el generar recursos humanos capacitados que permitan elevar la competitividad y producción nacionales e integrar exitosamente al país en la economía mundial; al mismo tiempo, que incorporen la sostenibilidad del recurso humano por medio de su formación integral. Se destaca que todo proceso de formación, capacitación y educación continua debe garantizar actividades sistemáticas de seguimiento que lo retroalimenten.

En los documentos citados, se establece la evaluación como un proceso que coadyuva en la valoración de la calidad del sistema educativo y a la integración de éste como un todo coherente. Dicha tarea se lleva a cabo desde las aulas de clase hasta las unidades administrativas más complejas. Para conducir este proceso, se utiliza una variedad de instrumentos que integra las valoraciones de los educadores y administradores, estudiantes, padres y madres de familia y la comunidad (local y nacional) sobre la calidad de los logros del sistema educativo en términos de conocimientos, procesos cognitivos y valores. 
Los presupuestos teóricos que rigen la "Política Educativa hacia el Siglo XXI”

En lo que respecta a la evaluación de los aprendizajes, la política señala:

- La necesidad de reflejar la coherencia entre el "qué" es esto, abordar en forma sólida los conocimientos; el "cómo", o sea, los procesos de construcción y reconstrucción de conocimientos y el "para qué" de la evaluación, es decir, las consecuencias desde el punto de vista de los valores.

- Debe verse como un instrumento para monitorear el aprendizaje, retroalimentar el proceso educativo y coadyuvar en la determinación de la calidad de la educación.

- Entre sus problemas están la carencia de indicadores y sistemas de evaluación que dificultan el establecimiento claro de objetivos y metas en la capacidad y calidad de la ejecución.

- Se dirige hacia el cumplimiento de los objetivos establecidos en los planes y programas. Por lo tanto, la respuesta a qué evaluar es la siguiente: los objetivos de cada área del saber que se encuentran en los planes y programas de estudio, teniendo el cuidado de que esos objetivos respondan a unos contenidos secuenciados y distribuidos gradualmente a lo largo del nivel escolar primario.
Desde el marco de referencia en que se circunscribe la evaluación, "la práctica educativa" la

constituyen los planes y programas de estudio; los textos y otros recursos didácticos, los procesos de evaluación, la mediación del docente y la formación. La capacitación y la educación continua de los educadores deben partir del énfasis en las disciplinas; en donde cada disciplina contribuya a la construcción y reconstrucción del conocimiento sin perder la identidad propia; para, finalmente, cuando sea apropiado, evolucionar hacia enfoques transdisciplinarios de convergencia en que cada disciplina complemente e ilumine a las otras. 
- Los criterios expresan el tipo y grado de aprendizaje que se espera que los estudiantes hayan logrado con respecto a los objetivos generales del área del saber, en cada ciclo.

- $\quad$ El marco jurídico que la respalda (MEP, 1994a; 1994b).

Desde el marco de referencia en que se circunscribe esta evaluación, "la práctica educativa" la constituyen los planes y programas de estudio; los textos y otros recursos didácticos, los procesos de evaluación, la mediación del docente y la formación. La capacitación y la educación continua de los educadores deben partir del énfasis en las disciplinas; en donde cada disciplina contribuya a la construcción y reconstrucción del conocimiento sin perder la identidad propia; para, finalmente, cuando sea apropiado, evolucionar hacia enfoques transdisciplinarios de convergencia en que cada disciplina complemente e ilumine a las otras (MEP, 1994a; 1994b).

El propósito de esta investigación es evaluar lo actuado por el PROMECE en el componente Evaluación Escolar. Con este propósito, hemos optado por un enfoque donde predomine la metodología cualitativa que interpreta y comprende la evaluación como un proceso complejo dentro de una perspectiva humana y social, que permite determinar conexiones reales (articulaciones) y rupturas (cortes) que se encuentran en una práctica educativa que posee diferentes planos, temporales y espaciales, como lo son las diferentes regiones y comunidades educativas (de Alba, 1994).

\section{Las preguntas investigativo-evaluativas}

Pocas veces se analizan, discuten y valoran los factores de éxito o fracaso, las lecciones aprendidas y los logros obtenidos por programas vigentes, desde la perspectiva de los actores sociales involucrados. La presente investigación se desarrolló en la Región Atlántica y consta de dos fases. Una de ellas se llevó a cabo con el Equipo Regional de Limón y la otra, con padres y madres de familia, estudiantes y docentes. En este caso particular, nos preguntamos

¿Cómo se valoran, desde una perspectiva cualitativa, los factores que coadyuvaron al desarrollo del componente Evaluación Educativa?

¿Qué significado educativo ha tenido para los actores y las actoras sociales (docentes, estudiantes, padres de familia, personal técnico-administrativo) el desarrollo del componente Evaluación?

¿Cuáles aprendizajes, individuales y colectivos se han logrado con el desarrollo de este componente? 
¿Qué acciones propondrían los actores sociales, a futuro para mejorar el desarrollo de la evaluación educativa?

\section{Los procedimientos metodológicos que permitieron la producción de la información}

La naturaleza de los problemas por evaluar, requiere combinar algunos elementos epistémicos que, desde un enfoque cualitativo, integre el trabajo de campo con el análisis, la discusión y la interpretación de los hechos que en su práctica educativa han construido actores y actoras sociales.

Para lograr los propósitos de la evaluación, se crea un clima de confianza mutua, que permite la empatía entre diferentes actoras y actores en la búsqueda de respuestas a las preguntas generadoras y a nuevos interrogantes que surgen en el desarrollo de la evaluación.

Con este proceso, se busca la flexibilidad para las aclaraciones necesarias sobre ideas, fenómenos, significados, símbolos, contradicciones, señales, normas, usos y costumbres educativas (cultura educativa), que vinculen la práctica con la teoría.

El Componente Evaluación Educativa incluye: pruebas nacionales para diagnóstico y para acreditación, de nivel y de ingreso. Es necesario incorporar en el proceso evaluativo a aquellas instancias que han tenido alguna responsabilidad en la toma de decisiones.

\section{Actores y actoras}

Entre los actores y las actoras que conforman la Región, participan en la evaluación:

- Un Director Regional.

- Un Jefe de Departamento Desarrollo Educativo.

- $\quad$ Cinco Supervisores / Asesores de Circuito.

- Dos Asesores Regionales. Uno de ellos, es el Asesor en Evaluación y el otro, un asesor vinculado con las materias básicas (Matemática, Ciencias, Estudios Sociales, Español e Idioma).

- En los talleres pedagógicos, participan 31 personas. Se realizó con él o la docente de aula, con 5 estudiantes de educación primaria, 11 estudiantes de educación secundaria, 2 padres y madres de familia, 8 docentes, padres de familia y alumnos de estos docentes, estos últimos fueron seleccionados por los y las docentes, considerando la disponibilidad para participar en el taller pedagógico. 
A partir de las preguntas evaluadoras, se utilizaron las siguientes técnicas:

- La entrevista focal, parte de los espacios de convergencia personal-profesional de carácter intersubjetivo que permite articular procesos. Con su aplicación, se busca explorar eventos relacionados con la ejecución y seguimiento de programas. Se usó principalmente en el equipo regional de la Zona Atlántica, ya que es una técnica consecuente con el tipo de labor que se realiza en esta instancia. Este proceso tuvo como guía las preguntas evaluadoras y la experiencia obtenida durante el proceso de investigación.

- El taller pedagógico, permitió reconstruir en forma participativa y desde la propia experiencia de los participantes, lo vivido en relación con la evaluación y proponer altemativas viables a las dificultades encontradas.

\section{La triangulación}

En esta evaluación, la triangulación se realizó con las diversas fuentes de datos; para ello se estudió y contrastó la situación evaluativa con los diferentes actores y actoras que participaban en la evaluación. También se utilizó la triangulación de técnicas, una vez realizados los registros de las observaciones y las entrevistas, al contrastar las diferencias y semejanzas que aparecen en la descripción y valoración de las distintas realidades educativas.

A continuación, se presentan los resultados obtenidos, en primera instancia desde el "Equipo Regional", y luego desde la información producida en los talleres donde participaron docentes, padres y madres de familia.

\section{VI. ¿Cómo analiza la práctica evaluativa el equipo regional de la Zona Atlántica?}

La entrevista grupal con el equipo regional, se desarrolló en el Colegio Técnico Profesional del Valle de la Estrella. En esta oportunidad, estuvo presente el asesor de evaluación de la región.

\section{La región y sus particularidades}

La preocupación principal del equipo regional radica en que no se consideran las particularidades de la región para construir las pruebas, en el sentido 
de adecuarlas a las características del contexto sociocultural, el tipo de estudiante, la ubicación del centro entre otros, ya que

"Cada vez que se acerca el proceso de evaluación para nosotros es dificilísimo, porque en Talamanca tenemos dos características, la bribri y la cabécar y a eso le aunamos que vienen pruebas donde nos preguntan que de Naranjo a Alajuela, San José tantas distancias... cuando el niño no conoce, bueno conoce en un mapa, personas que no han salido nunca de Limón... vienen en español" (ERA). ${ }^{3}$

Una de las críticas de que reiteradamente son objeto los contenidos de los instrumentos de evaluación, es la contextualización del lenguaje; al respecto conviene mencionar que la noción modema de validez (Messick, 1989. Moss, 1992), refiere a los aspectos contextuales de la medición y a las consecuencias sociales de la misma.

Además, para Habermas (1999), la acción comunicativa se sucede no por el cálculo de resultados, sino por actos de entendimiento. Un acuerdo comunicativo, debe ser aceptado como válido por los actores que interactúan en el proceso. El entendimiento requiere que "(a lo menos) dos sujetos lingüísticos e interactivamente competentes entiendan idénticamente una expresión lingüística" (pág. 393) .

La zona Atlántica se caracteriza porque sus habitantes son de origen afrocaribeño, donde la comunicación y el proceso inicial de socialización infantil es en el idioma inglés. También se encuentran otros grupos indígenas, así como los orientales. En lo que respecta al lenguaje utilizado, el asesor recuerda que

“en los hogares sólo en inglés se habla y cuando van a la escuela... vienen los niños también igual, es imposible que para que Limón pueda sacar un alto rendimiento a nivel nacional sino vemos todas esas singularidades que tiene el propio Limón, sí... Limón es multietnia y multicultural" (ERA).

Otra preocupación que expresa el equipo regional se refiere al bajo rendimiento académico de la región; una explicación a este fenómeno se expresa en el texto que se presenta a continuación:

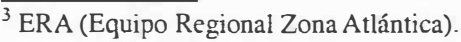


"quieren que nadie se gradúe, es la globalización... eso trae un trasfondo... quieren en cuatro años reducir el número de intelectuales a como haya lugar: Aquínecesitamos mano de obra barata, y ...¿ cuál es la mano de obra barata? Los que no se hacen bachilleres..." (ERA)

A la vez, el equipo regional percibe el apoyo del Asesor de Evaluación, de la siguiente forma:

"tenemos un excelente asesor de evaluación como lo dije al principio, es uno de los aspectos positivos que tenemos" (ERA).

\section{Los procesos de comunicación, ejecución y capacitación en la aplicación de las pruebas a nivel regional}

La comunicación y los procedimientos para la aplicación de pruebas se guían por el calendario escolar. Ante la pregunta sobre las formas de coordinación que sigue la región y las direcciones o departamentos del nivel central, la respuesta es que

“...yo sé el calendario escolar y punto, las pruebas son tal día y punto" (ERA).

La aplicación de las pruebas está calendarizada y las normas para ello se cumplen tal como emanan del nivel central.

El procedimiento que siguen para la elaboración y ejecución de algunas de las pruebas, es el siguiente

“...primero, hay una elaboración de ítemes previos para las pruebas. Para eso se mandan algunos asesores para que hagan los documentos. Los ítemes se mandan, se convalidan y luego vienen los temarios y después viene todo lo que es elaboración de exámenes, control de calidad los elabora... A la hora de ver la prueba uno se da cuenta [si aparece algún ítem mandado]... y a veces no aparece ningún ítem mandado... a veces aparece alguno, pero eso lo hacen por cumplir con un proceso" (ERA).

La participación de la región se concreta en el envío de ítemes. El proceso de aplicación de pruebas requiere de capacitación, y ésta se dirige 
“a los delegados, yo por eso dije en alguna oportunidad de sábados, ... yo he estado ahí cuando se me ha dado capacitación. Este año no, en el pasado tampoco y antepasado tal vez y a mediados... pruebas nacionales y haga la aplicación. Pero a nosotros los de abajo no tenemos ninguna opinión sobre eso... es que esa capacitación es para tirar exámenes" (ERA).

El proceso de aplicación de pruebas es catalogado como un procedimiento para "tirar exámenes". La participación en lo que respecta al contenido de la prueba no se concreta.

Por su parte, la aplicación del reglamento de evaluación conlleva una serie de aspectos legales que trasciende el ámbito escolar; el equipo regional considera que

“...tiene que jugar de abogado uno, aunque no lo sea. El comité de evaluación deberíamos tener una capacitación previa, para el colegio, nosotros no estamos preparados para manejar eso..." (ERA).

Para el Equipo Regional, la transición de la educación primaria a la secundaria, es un cambio drástico; en ese sentido,

"a mí me preocupan varias cosas, en primaria el alumno se trata de una manera, el maestro tiene una formación pedagógica amplia, buena, trata adecuadamente a sus alumnos con adecuaciones curriculares y eso y llegan (a secundaria) el profesor tiene 300 alumnos verdad, un horario, apenas si los ve... a veces se fueron los cuarenta minutos" (ERA).

En esta transición, los procesos de medición educativa para la educación secundaria y la educación primaria varían, por ejemplo

"cada proceso de sexto se maneja en un proceso aparte donde a veces yo creo que si los consideran noveno y bachillerato todo viene empacado, para noveno año viene con un seguro para que uno lo abra en el momento, y lo maneja San José, estos sí consideran más a los asesores y les consultan más" (ERA).

Otro ejemplo de comunicación que se vincula con la evaluación escolar se refiere a lo que el señor ministro llamó la "censura previa" 
“...el Ministro fue enfático dijo: no vamos a tolerar la censura previa, la prueba es responsabilidad del docente, si llega una apelación y el comité la revisó ya no es responsabilidad del docente, es responsabilidad del comité y ahí hay un comunicado directo por escrito, el Ministro nos está dando una orden... muchas veces se da el problema del poder con los comités evaluativos" (ERA).

"Los Temarios", tanto para el Bachillerato como para las pruebas de sexto, generan controversia y posiciones diversas. Las personas consultadas consideran que una buena estrategia para la ejecución de las pruebas es el temario

“...yo les digo en sexto trabajen con el temario, es un poco incorrecto pareciera pero el temario nos guía. Otra fortaleza es que don F. mandó con tiempo el cuadro de balanceo, cuando yo hice las pruebas vi cuáles eran los temas que más venían... eso a mí me permite que el maestro de sexto año le dedique más tiempo a los temas que más puntaje tienen en la prueba... espero que este año sea igual, que el cuadro de balanceo nos llegue a tiempo..." (ERA).

Sin embargo, otro entrevistado considera que el temario,

“...es una gran mentira, porque yo analicé el temario con los jefes de departamento del colegio técnico con el comité de evaluación y por lo menos en estudios sociales un ochenta por ciento de las preguntas no estaban, a nivel de bachillerato y a nivel de noveno mentira que lo toma"ron en cuenta..." (ERA).

\section{Algunos logros en el componente "Evaluación Escolar" desde la óptica de Equipo Regional}

La aplicación de las pruebas altera la rutina de las comunidades educativas. De ahí que los procesos que conlleva dicha aplicación sean vistos como de carácter centralizado y mecánico.

No hay duda que el órgano regional se ha preocupado por el bajo rendimiento académico de la zona, por ejemplo algunos circuitos escolares se han propuesto metas para mejorar esta condición y las estrategias para lograrlas. El equipo regional hizo referencia a un caso donde los alumnos se concentraron

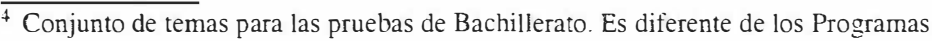


en una casa, pagada por la Junta de Educación. Los docentes se preocuparon de los centros de estudio, la alimentación, la recreación, entre otras. El resultado fue un ascenso significativo en el rendimiento. Otra estrategia que pusieron en práctica para mejorar el rendimiento fue la creación de

"un comité asesor que ...en octubre formuló las pruebas iguales, iguales como las hizo el MEP, con el mismo modelo, con las mismas características y las aplicamos, o sea, sacamos cuatro ratos para aplicarlas. Fueron maestros vecinos a aplicarlas, y nos dio resultados... vimos con tiempo los temas que estaban fallando aqui en el Valle y pudimos hacer un plan remedial y eso nos permitió sacar de un 90 hacia arriba" (ERA).

En el caso de una materia básica, como matemática, este educador que participa en el grupo señala que la prueba

"la hizo un grupo de profesores de matemática y la vi más pesada que la que yo hice aqui en el circuito... entonces yo creo que la idea es anticipando al proceso, hablándole y concientizando al maestro" (ERA).

Los aspectos logísticos relacionados con la aplicación de las pruebas que emanan del Ministerio de Educación, se valoran positivamente, porque

"todos los docentes, por lo menos en mi circuito, tienen su reglamento; todos los docentes tienen sus registros para llevar el control de pruebas, a todas las instituciones de dirección dos para arriba se les integra un comité de evaluación que es remunerado, en mi circuito revisan las pruebas como política institucional ...es mejor prevenir que curar" (ERA).

El recurso humano calificado, en este caso con un posgrado en evaluación educativa, ha procurado cambios en la región; el grupo entrevistado considera que

"casi todo el mundo fue formado para evaluar contenidos yo creo que ahí está un divorcio fuerte, M... [asesor en evaluación] ha venido a cambiar ese sistema pero todavía le falta a los maestros, en teoría muy bien pero aplicarlos cuesta" (ERA).

El acercamiento de los padres y las madres de familia a las instituciones es motivado, en la mayoría de los casos, por el rendimiento académico de sus hijos, ya que 
"a ellos [los padres] lo que les interesa del alumno es la evaluación, no si está siendo bien atendido, si está mal atendido, el padre de familia lo que llega al colegio es a preocuparse si mi hijo va bien, el padre de familia se atiende en esos aspectos. El maestro está documentado para eso, por lo menos en eso nos preocupamos creo que los nueve asesores que hay en la región para estar al día en eso, ... viera que feo si llega un padre de familia a reclamar y no se le puede contestar nada, esos son los aspectos positivos de la evaluación" (ERA).

La aplicación de pruebas genera una dinámica, propia de cada cultura evaluativa, porque involucra a diferentes actores y actoras, quienes se desenvuelven de acuerdo con procedimientos administrativos; pero al mismo tiempo, con prácticas propias de cada región. Los "temarios" generan diversidad de opiniones, que van desde ser considerados una guía hasta un poco de incertidumbre. La aplicación de pruebas deja entrever diversas preocupaciones por aspectos legales que podrían afrontar los y las docentes.

\section{Algunos obstáculos encontrados en las prácticas evaluativas oficiales}

Los entrevistados y las entrevistadas expresan sus lógicas con respecto a la estandarización de las pruebas versus la calificación del recurso humano; por ejemplo, menciona que:

"no se toman en cuenta las diferencias individuales cuando hay una ley que dice que tenemos que ser iguales. Cuando yo tenga todos los profesores MT-5 hágame la misma prueba MT-5 para todos, pero usted no me puede evaluar en sexto grado una escuela allá de Gavilán con la escuela Perú de San José y es la misma prueba de sexto grado, vienen de San José" (ERA).

La situación anterior se une a la falta de permanencia, o bien, a la ausencia del recurso humano calificado, porque

“...todo eso viene a dar con los problemas de nombramientos, la falta de plazas para el personal de secundaria. Aquí vienen muchos profesores que concursan la plaza para entrar en propiedad, a veces no hay personal o por otros vicios. Hay problemas serios de nombramientos... un curso lectivo no tuvo profesor de español ¿cómo va ir un muchacho a bachillerato así...?" (ERA). 
A la vez, funcionarios y funcionarias abogan por la regionalización de las pruebas, como lo ha sido en experiencias previas, ya que

“...allá en San José, dicen que Limón tiene bajo rendimiento, no... déjenos hacer las pruebas como las hicimos el primer año, con nuestros maestros de sexto grado, con nuestros asesores, ¿por qué tienen que mandarnos alguien de San José a evaluarnos al Colegio de Talamanca o al Colegio del Valle de la Estrella, y lo están evaluando igual que el Liceo de Costa Rica o el San Luis Gonzaga de Cartago?..." (ERA).

Actores y actoras perciben cierta desconfianza dirigida a su desempeño en tareas que consideran, pueden realizarse desde la región, porque

"desconfían de los profesores y de los maestros, tenemos que rescatar esa confianza. ¿De dónde se ha fugado esa información es del Valle Central? ...gracias a Dios que nunca han dicho Limón. Ustedes saben a qué me refiero pero sí tenemos que rescatar esa confianza del profesor que sea capaz de hacer una prueba con honestidad y que es confiable" (ERA).

El nivel regional percibe una falta de coordinación entre la División de Control de Calidad y el Departamento de Evaluación, esta situación trasciende al ámbito conceptual del campo disciplinar, debido a que

"...el que tiene que evaluar es el Departamento de Evaluación del Ministerio, no control de calidad. La palabra lo dice "control de calidad" es para controlar no para evaluar. Ellos quieren toda la pelota con el visto bueno de no sé quién... si será el Consejo Superior, si será el Ministro. Ellos hacen y deshacen... (ERA).

Se reconoce el papel que cumple el comité de evaluación al considerarlo como un acto de coaprendizaje, en el sentido que

“...hay muchos docentes que no tienen la experiencia todavía en el campo de la formulación de ítemes y el trabajo en equipo fortalece, evacuando dudas y se ayuda al compañero..., y hay veces que el niño no comprende, entonces, al quitar la revisión grupal del comité de evaluación, no se está fortaleciendo esa área..." (ERA).

El equipo regional aboga por incentivos para quienes desempeñan el cargo de Directores 1 y los unidocentes, al señalar que 
“...una parte negativa que yo veo en la evaluación, los directores uno y los unidocentes no se les paga. Ellos tienen lecciones técnicas y administrativas, ellos forman comités, se desplazan a distancias, no se les paga el incentivo... no hay ningún incentivo salarial..."(ERA).

Otros aspectos legales interfieren en decisiones técnicas que toma el docente; por ejemplo, un docente

"le aplicó una acción correctiva a un alumno, la madre se fue donde el abogado del patronato y le mandó esto, con artículos y todo, eso es ilegal de acuerdo con el código de la niñez, el asesor me dio la razón,... quedó sin efecto la acción correctiva, ... hay que apegarse a eso porque es una ley" (ERA).

Con respecto a los redondeos de las calificaciones y sus respectivos centésimos, es que se decide si la pregunta está mala o está buena y, por consiguiente, la suerte de los estudiantes. La promoción, a veces, la deciden las máquinas. Esta posición la explica un funcionario, de este modo:

“la máquina es fría, la máquina no piensa, la máquina agarró un papel y dijo bueno o malo... a esta oración de pasar del singular al plural le faltó una "ese" solo porque a la palabrita le faltó una "ese"no le voy a poner todo malo, la máquina se la pondría toda mala, ... ustedes lo han visto en las actas oficiales, por centésimas se queda el muchacho, porque la máquina es fría" (ERA).

Las apelaciones producto de las pruebas, también son motivo de preocupación por parte del equipo regional, ya que

"De acuerdo con la Ley cada dirección regional debe tener un abogado, solo en San José se los presta a medio tiempo el Colegio de Licenciados y Profesores, pero en ninguna dirección regional hay un abogado,... que se nombre un abogado que nos ayude en las regiones para evacuar dudas... montones [de apelaciones] pero se resuelven en cada oficina de supervisión...” (ERA).

Un obstáculo que se ha encontrado es el tiempo adicional de dedicación a la evaluación. La función de la evaluación como parte de la enseñanza se ha desvirtuado, porque 
"al maestro no se le paga para evaluar, al maestro se le paga para ensenar, la evaluación es parte del proceso y a veces los profesores y los maestros lo toman como lo principal... ¿entonces qué? nos vamos a pasar solo en esas... del rendimiento" (ERA).

El texto anterior induce a la reflexión y a plantearse las preguntas ¿En procura de qué hemos dedicado tanto esfuerzo a la evaluación? Y los resultados de la medición, ¿se han traducido en mejorar el sistema educativo en general y al aprendizaje en particular?, ¿qué investigaciones se desarrollan con los resultados de las pruebas nacionales?, ¿qué papel juegan los y las docentes en todo este proceso?

\section{¿Qué propone el equipo regional?}

El equipo regional propone fundamentalmente un cambio: la regionalización de las pruebas. Considera que se puede modificar el procedimiento de elaboración de pruebas. Esta posición la expresa ese equipo de trabajo de la siguiente manera:

¿Por qué no darle recursos a la región para que realice las pruebas o elabore las pruebas de acuerdo con su particularidad? ...bueno hacer el balance por región, no podemos permitir un criterio así, a nivel nacional... ¿dónde vamos a meterlos a todos? ¿en el mismo canasto? ... sí somos iguales... pero somos particulares también... ahi es donde está el error de las pruebas de sexto y de noveno... Claro que necesitamos asesoría, necesitamos apoyo en ese sentido... las pruebas deben ser regionalizadas... (ERA).

Actores y actoras confían en su capacidad técnica para cumplir con los procedimientos que exigen las pruebas. Esa capacidad la plantean de la siguiente forma:

Los profesores de la región de Limón [son] capaces de poder hacer una prueba de bachillerato. En Limón existen, en Guápiles, Puntarenas, en todas las regiones hay equipo regional muy bueno, a nivel de sexto también... demostrarles que somos capaces y que tenemos el equipo suficiente de hacer pruebas aqui a nivel regional..." (ERA).

También consideran que el proceso evaluativo debe ser apoyado por un curriculista a nivel de circuito; tal como se expresa en la siguiente cita: 
“...por lo menos un recargo, un recargo por circuito y que asesorado por [el asesor de evaluación], por lo menos que le paguen un 50\%...” (ERA).

Las propuestas para mejorar el rendimiento de los colegios de la región van unidas a estrategias como las siguientes:

“...el Colegio del Valle para mí es un ejemplo, pasó de ser un colegio con muy bajo rendimiento a tener un $100 \%$ en bachillerato y ...de los $27 \mathrm{mu}$ chachos que hicieron bachillerato solo se quedó una muchacha en español ¿qué hicieron? Me di cuenta que este colegio hizo toda una estrategia, por ejemplo, un detalle, hay muchos muchachos pobres que tienen problema para comprar la calculadora científica, el colegio compró las 27 calculadoras científicas y las tiene en la biblioteca..." (ERA).

La visita al colegio que se menciona en el texto anterior, permitió conocer una serie de estrategias que siguen docentes, personal administrativo y las familias para lograr subir el rendimiento a nivel de bachillerato. Por ejemplo: la Junta de Educación alquila una casa donde los y las jóvenes se concentran. Ahí reciben apoyo académico, recreativo, cuidan de su dieta. Eso exige trabajo docente fuera de horas laborales, que funcionarios y funcionarias han cumplido con el único incentivo de mejorar los resultados de las pruebas de bachillerato.

\section{¿Cómo utiliza el equipo regional los resultados de las pruebas?}

El equipo regional comunica que antes de 1994, no se encuentran informes estadísticos que muestren la situación de rendimiento académico; sin embargo,

"por lo menos se les dio la información estadística hasta por circuito, cuántos aprobaron, cuántos reprobaron, ...Nosotros en Limón, por lo menos en primaria, estamos arriba de la media nacional... es un orgullo para nosotros verdad... En el plan del 2000, también nosotros hemos influido ahi [para] mejorar el rendimiento a nivel regional...".

Los resultados de las pruebas son analizados tanto por el equipo regional como por directores y directoras de instituciones educativas. Esta información se discute de diferentes maneras, tal y como puede observarse en el siguiente texto: 
"nos llega la información y entonces la analizamos a nivel de directores ¿qué hacemos para mantenerlo o qué hacemos para mejorarlo...? a veces el asunto no es tan bonito, a veces tenemos un rendimiento del 70\%; entonces ahi nos sentamos, conversamos lo analizamos y nos comprometemos con nosotros mismos a mejorar esa situación" (ERA).

En el circuito 07 de Talamanca, los resultados se analizan tal y como se menciona a continuación:

“...analizamos con cada institución, nos sentamos y hacemos un plan estratégico de mejoramiento en las diferentes materias... muchas veces la culpa no es del alumno. Nos hemos dado cuenta de que el docente le tiene miedo a algunas materias, ... ¿por qué no se asesora cómo dar ciertos temas, ciertos contenidos programáticos? y ...lo que hace es que sencillamente no los da. Hacemos un plan de mejoramiento y traemos las personas para que den los talleres que se necesitan en los circuitos..." (ERA).

En la región, se insiste en mejorar los índices de calidad de la educación primaria especialmente en matemática y estudios sociales que:

"andamos relativamente bien; pero este año, no hemos recibido la información estadística de la promoción general por asignaturas, por centros educativos en orden descendente, uno tomaba en cuenta eso para hacer las visitas a los centros en el caso de primaria..." (ERA).

En este colegio de secundaria, el equipo regional ha tomado las siguientes medidas:

"hemos andado más mal... hacer un plan integral, atacar el asunto con el padre de familia, para que colabore... más visitas... replanteaba las estrategias en el aula... eso nos dio resultados, fuimos creciendo lentamente. Este año ha habido un descontrol porque nombraron un director, hizo el periodo de prueba y se fue... estuvo el colegio sin director y no hemos podido dar seguimiento a eso..." (ERA).

De nuevo, la movilidad del personal docente y administrativo, incide en el desarrollo de procesos que requieren de seguimiento.

La entrevista realizada al equipo regional, permite señalar que las preocupaciones se centran en la necesidad de regionalizar la confección y aplicación de 
pruebas, que hasta ahora poseen carácter nacional. El equipo regional confía en los recursos humanos calificados con los que cuenta para que el proceso sea responsabilidad de la Región. Actores y actoras manifiestan inquietudes en lo que respecta a los procedimientos establecidos para dicha aplicación, la capacitación que se requiere y los aspectos legales implícitos.

\section{El proceso evaluativo desde la perspectiva de estudiantes, docentes, padres y madres}

La técnica utilizada en esta fase fue el "Taller Pedagógico" que se desarrolló en la Escuela Los Corales en la ciudad de Limón. Asistió un total de 20 estudiantes ${ }^{5}$ provenientes de la Escuela Líder Los Corales, de los Colegios Técnico Profesional Valle de la Estrella, Colegio del Río Banano, C.P.I de Limón; además, dos padres y cinco madres de familia. También ocho docentes, dos de ellas Directoras de Escuela y las otras seis, se desempeñan como docentes ${ }^{6}$.

En la primera parte del Taller, se trabajó en grupos, es decir, hubo un grupo constituido por estudiantes, otro por padres, madres y docentes, y expresaron, metafóricamente, su vivencia evaluativa. En la segunda parte, se realizó una puesta en común.

Los niños y las niñas más pequeños denotaron su concepto de evaluación con unos dibujos explicativos. La explicación se transcribe a continuación.

\section{Lo que pensamos de la evaluación ${ }^{7}$}

"En este dibujo hay un niño que le fue bien en el examen" (el niño se nota alegre).

"Este dibujo presenta un niño con el examen que se sacó un 20 porque estaba un poquito más duro" (el niño se nota triste).

"Él volvió a hacer el examen y la calificación fue de cien, está pensando que la mamá se iba a poner mucho más alegre y que lo abrazaría y le daría muchas felicitaciones".

5 Participaron 11 estudiantes de educación secundaria y nueve de educación primaria.

${ }^{6}$ Las manifestaciones verbales de ellos y ellas se transcriben tal como fueron expresadas.

${ }^{7}$ Es una transcripción textual de lo expresado por niños y niñas durante el taller. 
"Lo que quiere decir esto es que algunos niños al hacer el examen vienen pensando si se lo sacan malo llegan a la casa y los van a regañar o si se lo sacan bueno se van a sentir orgullosos".

"Este niño fue que él lo hizo y la calificación se la sacó muy mala, él fue pensando poco a poco y él pensó que le iban a pegar después de eso la mamá lo aconsejó y le ayudó a poder estudiar".

Los once estudiantes de secundaria representaron su noción de evaluación por medio de una balanza, cuyo peso se va hacia el nivel de la evaluación. La han representado con las siglas BV (bolsa de valores) que pesa más. Al otro lado de la balanza, colocaron un libro.

En cuanto a la representación anteriormente citada, los estudiantes la explican textualmente tal y como aparece a continuación

“...hay un desnivel ahora se nos exige más en cuanto a capacidad y rendimiento. La balanza diferencia lo bueno y lo malo que como estudiantes tenemos. También representa la diferencia en cuanto a materia de evaluación que exige el Ministerio porque ellos quieren que solo pase lo mejor".

Padres y madres de familia que participaron, connotaron la evaluación utilizando tres refranes que se explican textualmente, a continuación

\section{Con la vara que midas serás medido}

"Podemos comparar la evaluación porque de la misma forma en que los docentes califican a los estudiantes, también los mismos educadores están siendo calificados por los directores, por los padres de familia,... entonces las evaluaciones tienen que ser de una forma objetiva".

\section{Ayúdate que yo te ayudaré}

“...este caso se da entre los alumnos que tratan de sobresalir que son buenos estudiantes, algunos que les cuesta y tratan de no preocuparse para tratar de alcanzar una nota pero muchas veces ellos no pueden. En este momento es donde el profesor debe saber ¿cuál es el estudiante que le cuesta? y ¿cuál es el estudiante que en realidad saca notas (bajas).... porvagancia? Entonces, el alumno que uno ve que le cuesta, que quiera 
alcanzar la nota mínima que es un 65, en este momento el profesor debe de ayudarlo. Hay un momento en que el alumno no puede más, es ahí donde el profesor debe ayudarlo todavía con mucha más razón a quienes están pasando alguna dificultad...".

\section{El que persevera alcanza}

"Aquí es donde el estudiante se propone metas, si todo estudiante se propone metas llegará a ser algo en el futuro, si persevera logrará alcanzar lo que se propone en su vida."

Las cuatro docentes que participaron en el taller representan la evaluación como una escalera.

\section{La evaluación como una escalera}

"Vemos la evaluación como una escalera, cada peldaño es un avance, conforme nosotros vamos avanzando en lo que es evaluación nosotros vamos adquiriendo más conocimientos. Si nos quedamos solamente en un peldañito volvemos a repetir los mismos errores una y otra vez hay que avanzar, hay que seguir adelante”.

También expresaron sus vivencias por medio de lo que les reporta la experiencia.

\section{Más sabe el diablo por viejo que por diablo}

“Mientras más sepamos de evaluación más preparados estamos para poder aplicarla, eso de la experiencia es que mientras nosotros apliquemos diferentes métodos y utilicemos diversas cosas entonces nosotros vamos a tener más experiencia para ver qué es lo que nos sirve y qué es lo que no nos sirve en ciertos alumnos".

El otro grupo de docentes que participó en el taller, representa la evaluación por medio de estos refranes

\section{Con la vara que midas serás medido y una cuarta más}

"Representa aquí la evaluación como nosotros la pensamos es solamente calificación, rendimiento, o sea entre más duro el maestro mejor va a 
ser el estudiante, mejor que le toque con la niña tal porque esa sí es estricta, sí es dura, es quebradora. En el colegio el profesor que tiene más fama es el más quebrador, el que a todo mundo deja y el más malo es al que todo mundo le pasa."

Las representaciones permitieron reconocer la preocupación de los estudiantes de primaria. Esto evidenció el estado de ánimo y la actitud de los padres frente a las notas. Las y los jóvenes de secundaria simbolizaron la evaluación con una balanza, que se inclina hacia los mejores estudiantes. Los padres y madres de familia trasladan la noción a la justicia y la solidaridad entre estudiantes y entre docentes. Los y las docentes por su parte, clarifican medición y evaluación y cómo son valorados socialmente por el número de estudiantes que pasan o reprueban.

\section{¿Cómo viven actoras y actores el proceso evaluativo?}

- Las y los estudiantes

Alumnos y alumnas de secundaria se preparan para los exámenes preferiblemente con tiempo suficiente para estudiar, de la siguiente manera

"antes del examen estudiando, evacuando dudas, hacer repasos, centros. Durante el examen tratar de no presionarse y estar muy seguros de lo que respondemos. Estamos en desacuerdo en que los temas que nos den los profesores sean una semana antes porque hay profesores que digamos lo llaman a uno y... el lunes... para que la prueba sea el martes, yo creo que esa es mucha presión para nosotros" (TPA) ${ }^{8}$

- $\quad$ Padres y madres.

Los padres y madres que participaron en el taller tratan de

"repasar con los hijos la materia, revisar los cuadernos para asegurarnos que tengan la materia completa, asegurarnos de que llevan los implementos necesarios para los exámenes como el lápiz, lapicero, borrador. En lo que no estamos de acuerdo es cuando hay demasiada materia para una sola prueba" (TPP).

\footnotetext{
8 TPA (Taller Pedagógico Alumnos).

9 TPP(Taller Pedagógico Padres).
} 
Padres y madres manifiestan su desacuerdo cuando se reprograman los días de evaluación y recomiendan que en un solo día haya una prueba para una misma asignatura.

Como es de esperar, los docentes participan antes, durante y después de la evaluación en las siguientes tareas:

- $\quad$ Aplicar, revisar y calificar pruebas.

- Estructurar cuadros de balanceo y pruebas.

- Confeccionar la prueba con base en los objetivos.

- Retroalimentar el proceso con base en los resultados.

A los docentes les interesa:

- Conocer el nivel de aprendizaje que logran los alumnos.

- Conocer el nivel de calidad de enseñanza que imparte como educador.

Educadores y educadoras no están de acuerdo con:

- La ejecución tradicional y metódica de las pruebas.

- La capacitación sólo para realizar pruebas tradicionales.

- Ciertos comités que no transmiten la información correcta a sus compañeros.

- El incumplimiento de ciertos docentes que no respetan las fechas indicadas para la aplicación de las pruebas.

Las directoras consideran que les queda poco tiempo para retroalimentar el proceso evaluativo, ya que

"el proceso de evaluación en las escuelas es simple y sencillamente estadístico. Nosotros nos interesamos por el proceso de evaluación para saber, cómo están los segundos años, cómo está el primer año, cuántos chiquitos se van a promover, cuántos van a ir a pruebas de aplazados. Para efectos estadísticos damos seguimiento y en el caso de los docentes antes y durante las pruebas porque ellos incurren en un proceso mucho más grande, ... y luego si nos queda tiempo retroalimentar lo que no está bien, de la información que nos da la evaluación" (TPD) $)^{10}$.

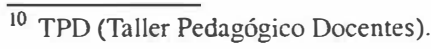




\section{El valor social de la evaluación}

La evaluación educativa adquiere valor social en la medida que es constituida por constructos creados socialmente, que son los que la van definiendo. De este modo, la idea del consenso social que prevalece, es la que nos hace aceptarla y pensar que no puede ser de otro modo (Álvarez, 1995).

Estudiantes, docentes, madres y padres coinciden en que la evaluación:

- Mide el grado de conocimiento adquirido durante el curso.

- Les permite desarrollar confianza, seguridad, capacidad, confianza consigo mismos (estudiantes).

- $\quad$ Permite conocer el grado de aprendizaje y modificaciones de la conducta del y la estudiante con base en los objetivos propuestos.

- $\quad$ Permite comprobar el rendimiento.

\section{La ruta que siguen los resultados de las pruebas nacionales}

Actoras y actores del proceso, inician con una fase explicativa de lo sucedido. Posteriormente, las situaciones vividas se archivan. Durante la aplicación de la prueba aparecen problemas, más que todo vinculados a la prueba en sí y a su contenido.

\section{La fase explicativa de los resultados y el respectivo archivo}

Cuando los resultados de las pruebas son entregados a los y las estudiantes, se preocupan por las explicaciones que les pueda brindar el o la docente, por ejemplo:

"que nos hagan un repaso de todo el examen porque digamos uno contesta algo y la tuvo mal y uno no sabe por qué, pedirle al profesor que nos diga ... ustedes tienen esto malo por esto y esto... Los resultados los archivamos para que más adelante nos sirvan de información" (TPA).

Los padres y madres también archivan los resultados una vez que han revisado las pruebas, para ver en cuáles asignaturas fallaron sus hijos o hijas.

Los resultados de las pruebas son utilizados en la institución de este modo:

- $\quad$ Es un instrumento de autoevaluación para las y los docentes.

- Brinda información institucional: estadística, control, promoción a todo funcionario administrativo. 
- Diagnostican el rendimiento, proporcionan una perspectiva general de grupo a los y las docentes.

\section{Los problemas encontrados y sus soluciones}

Los y las estudiantes manifiestan que se les asignan trabajos de investigación durante la semana de evaluación al mismo tiempo que les imparten materia nueva, ante esto:

“...planteamos el problema al profesor, para llegar a un acuerdo, [acomodar] el horario personal... nos imparten materia nueva, sin haber evaluado antes la prueba correspondiente a la materia anterior" (TPA).

Los docentes encuentran como problemas:

- $\quad$ Ítemes de las pruebas mal planteados.

- $\quad$ Puntajes mal calculados.

- Instrucciones que no se especifican claramente.

- Utilización de vocabulario desconocido para estudiantes.

- Inseguridad en los docentes debido a la falta de capacitación.

- Los y las estudiantes duran más tiempo del programado, cuando responden la prueba.

- Niños y niñas no reciben orientación individualizada cuando se les entrega la prueba.

La preocupación básica de padres y madres coincide con la expresada por los y las docentes en lo que se refiere a la utilización de vocabulario desconocido que aparece en las pruebas.

\section{Las pruebas nacionales}

Los y las estudiantes consideran que en las pruebas

"los evalúan muy injustamente porque en el caso de noveno dicen que si no ganan las pruebas no pueden pasar y si no tienen una nota de 70 en los exámenes no pueden hacer los exámenes. Entonces pensamos que eso es algo muy injusto" (TPA).

Todos sugieren: 
- Eliminar la nota de presentación

- Volver al método antiguo en cuanto a pruebas de noveno

- Cambiar el tiempo entre cada prueba (podría ser de día por medio)

Padres y madres asumen una posición crítica en lo que se refiere a las pruebas

"son buenas, pero no son justas en el sentido de que se hacen muy general a través de todo el país, sin importar las condiciones que tienen por ejemplo las instituciones rurales, que se encuentran en desventaja en comparación con las instituciones privadas o las del centro de la capital" (TPP).

Los padres y las madres sugieren que las pruebas se realicen a nivel regional y solicitan que los recursos no sean tan limitados.

\section{Desde el punto de vista de los docentes y directores:}

- $\quad$ Las pruebas deben regionalizarse.

- La prueba de sexto grado es otra medición de lo que ya fue medido.

- La prueba debe ser más formativa y desarrollar más la sensibilidad del ser humano.

\section{Los cambios señalados por los y las estudiantes de primaria apuntan a} que:

- Los exámenes sean más claros, porque lo ponen todo pegado.

- Las palabras que ponen en los exámenes no son comprensibles.

- Las maestras en vez de hacer siempre exámenes, repartan temas en grupos para desarrollarlos en la casa y después llegar a la clase a presentarlos, también hacer maquetas y explicarlas.

Los cambios sugeridos por los y las estudiantes de secundaria, proponen que:

- La directiva no se inmiscuya en lo que a evaluación se refiere.

- La directiva sólo se ocupe de la organización y realización de proyectos.

- $\quad$ El porcentaje de la nota que pone la directiva, se traslade a los profesores. 
Los cambios planteados por padres y madres de familia, plantean que:

- Haya más equidad entre las pruebas escritas y las orales.

- Los educadores tengan más vigilancia y asesoramiento en materia de evaluación.

- Se dé más valor a la participación oral y que los docentes les enseñen a expresarse mejor.

\section{Los cambios que proponen los docentes y las docentes son los siguientes:}

- Incluir ítemes que contribuyan al fortalecimiento de los valores.

- El y la estudiante no se deben sentir encasillados y han de tener más libertad para interactuar.

- Adecuar la prueba a la región geográfica.

- Elaborar una prueba diferente para los colegios técnicos.

- Estudiar la asociación prueba-temario.

- Subir el valor del trabajo cotidiano.

\section{El análisis de la información producida por el equipo regional conduce a los siguientes hallazgos:}

1. A pesar de que se reconocen particularidades propias de cada región, no se realizan adecuaciones en el Sistema de Evaluación por la existencia de un reglamento único de Pruebas Nacionales.

2. Los equipos regionales se han preocupado por poner en práctica estrategias innovativas que les han ayudado a mejorar el rendimiento escolar en la región, especialmente en aquellas instituciones que presentan bajo rendimiento.

3. La participación de asesores regionales y docentes se ha hecho efectiva en la confección y revisión de ítemes. En aquellas regiones donde se cuenta con un Asesor de Evaluación, especialista en la materia, la conducción de la práctica evaluativa se ha visto fortelecida.

4. En la Región Atlántica, se reconoce que el Programa de Pruebas ha llevado al Ministerio de Educación Pública a organizar los procedimientos de apoyo logístico, que influye en el desarrollo de dicho Programa.

5. Como consecuencia del Programa de Pruebas, la labor del Asesor de Evaluación se fortalece con ayuda de los asesores específicos. Esto pese a la incongruencia señalada entre las directrices de Control de Calidad y del Departamento de Evaluación. 
6. El contacto que mantienen los miembros del Equipo Regional con diferentes actores y actoras educativos, les proporciona una perspectiva real de la práctica educativa, respecto a los técnicos y autoridades del nivel central.

7. Los obstáculos que visualizan los equipos regionales, se refieren a la aplicación de Pruebas Nacionales en instituciones con modalidades educativas diferentes, así como a centros educativos con condiciones de funcionamiento desigual. Muchos de los problemas que enfrentan se originan por la presencia de docentes aspirantes, cuyo paso por la zona es circunstancial.

8. El énfasis de la evaluación centrado en contenidos, no es coherente con los fundamentos de la Política Educativa, pues no considera los aspectos constructivistas y humanistas ahí señalados, así como el desarrollo integral de los educandos.

9. Hay incongruencia entre la evaluación del aula orientada por el Reglamento de Evaluación, con las Pruebas Nacionales que incluyen sólo un tipo de pregunta.

10. Los y las docentes muestran preferencia por la medición y la aplicación de exámenes; sin embargo, se sienten inseguros para medir y evaluar utilizando otras técnicas altemativas.

11. El énfasis que el Sistema Educativo le ha dado a la medición y la evaluación educativa, ha provocado que tanto docentes como equipos regionales, le asignen excesivo tiempo y esfuerzos a esta función.

12. Los equipos regionales analizan los resultados de las pruebas y con base en ellas, generan acciones de apoyo a los docentes y las docentes, tanto por asignaturas, como por áreas y circuitos.

13. Los Comités de Evaluación son altamente valorados, porque generan procesos de coaprendizaje y coevaluación entre docentes, constituyendo un canal de intermediación con los padres y madres de familia; a la vez, sirven de puente con la Asesoría de Evaluación en el Nivel Regional.

14. Los temarios, en gran medida, han venido a sustituir los Programas de Estudio, en detrimento de la propuesta curricular vigente.

\section{El análisis de la información producida en los talleres pedagógicos condu- ce a los siguientes hallazgos:}

1. La representación del concepto de evaluación por parte de diferentes actores y actoras, se asocia con vocablos que denotan: "previsión", "desbalance", "trabajo excesivo", "lucha", "perseverancia y metas" entre otros. 
- Los educandos se inclinan por una representación positiva, que implica esfuerzo y reconocimiento.

- Los padres y las madres de familia abogan por la justicia, el apoyo a hijos e hijas, que implica un recargo en su trabajo cotidiano y la perseverancia.

- Los y las docentes lo asocian con exceso de trabajo, experiencia, previsión y esfuerzo.

2. El valor social de la evaluación es reconocido, tanto en el ámbito personal como colectivo, para el desempeño y logro de los aprendizajes e indirectamente para valorar el desempeño docente.

3. Los resultados de las pruebas son utilizados en forma diferente por diferentes actores y actoras.

- A los educandos, les permite cerciorarse de un aprendizaje y tomar decisiones para la próxima prueba.

- Para los padres y madres de familia es un motivo de alegría o de preocupación.

- A los y las docentes les permite diagnosticar, analizar y corregir. También se convierte como un instrumento de Autoevaluación. No hay evidencia de que los resultados se trasladen efectivamente a la institución y al currículo para realizar los cambios necesarios.

4. Con respecto a los problemas

- Los y las estudiantes enfrentan problemas con la confección de las pruebas que incluye vocabulario desconocido, materia no explicada por el o la docente y errores en la confección. Adicionalmente, reclaman por la asignación de trabajos extraclase y materia nueva.

- Los padres y madres coinciden con la opinión de los estudiantes.

5. Con respecto a las soluciones:

- Los y las estudiantes señalan que es conveniente hacer centros de estudio para relacionar y adecuar contenidos, capacitarlos en técnicas de aprendizaje y elaborar mejores pruebas.

- Los padres y madres de familia hacen alusión a considerar el diálogo entre docentes y el Comité de Evaluación. En síntesis, hacer uso adecuado de las diferentes instancias para el trámite de apelaciones. 
- Los y las docentes opinan que necesitan más asesoramiento, acudir al Comité de Evaluación y al Consejo de Profesores. También hacer reuniones con padres y madres de familia y orientadores, profesores y profesoras guía y estudiantes. 


\section{BIBLIOGRAFÍA}

Álvarez, J. M. (1995). "Valor social y académico de la evaluación". En Volver a Pensar en la Educación. Prácticas y Discursos Educativos. Vol. II (pp. 173-193)

Angulo, J. F. (1995). "La evaluación del sistema educativo: algunas propuestas críticas al porqué y al cómo". En Volver a Pensar en la Educación. Prácticas y Discursos Educativos. Vol. II (pp. 194-219) Madrid: Ediciones Morata.

Banco Interamericano de Desarrollo (1998). Evaluación del marco institucional del sistema educativo de Costa Rica: Informe Final. Washington D.C., agosto.

De Alba, A. (1991). Evaluación Curricular. Conformación conceptual del campo. México: Universidad Nacional Autónoma de México.

De Alba, A. (1996). Análisis de discurso educativo curricular. Una propuesta de evaluación curricular. Memoria. Foro-Taller sobre Tendencias Actuales en la Medición y Evaluación Educativa. Maestría en Evaluación Educativa: Universidad de Costa Rica.

Habermas (1999). Teoría de la acción comunicativa l. España: Taurus.

Ministerio de Educación (1996). Informe Consolidado. Resultado de la Evaluación de medio período. Programa de Mejoramiento de la Calidad de la Educación. Costa Rica.

Ministerio de Educación (1994a). Política Educativa hacia el siglo XXI. Costa Rica.

Ministerio de Educación (1994b). Marco de Referencia para una Política Educativa Nacional. Sintesis de una concepción del proceso educativo para la orientación de la Evaluación. Despacho del Ministro de Educación. Costa Rica.

Ministerio de Educación (1986). Programa de Mejoramiento de la Calidad de la Educación. Costa Rica.

Ministerio de Educación (1998). PROMECE, Informe de Avance.

Ministerio de Educación. "Anexo 3A", s.f. Costa Rica.

Ministerio de Educación (1991). Componente de Desarrollo Profesional, Proyecto MEP. BID. BIRF. Costa Rica.

Ministerio de Educación (1996). Convenio Formación de Docentes MEP, UCR, UNA, UNED, Plan de Acción, Antecedentes y Proyecciones.

Ministerio de Educación (1995). EL RETO DEL TERCER MILENIO: Una propuesta de un Proyecto Educativo Nacional Hacia el 2005 (EDU 2005), noviembre de 1995. Costa Rica.

Ministerio de Educación Pública (1999). Plan Estratégico para la Excelencia y la Equidad de la Educación, separata.

Picado, Marta (1997). Resumen Ejecutivo del Componente Evaluación, San José: Ministerio de Educación, noviembre.

Putnam, L. E. (2004). "La población afrocostarricense según los datos del censo de 2000”. En: Rosero, L. (Ed.). Costa Rica a la luz del censo 2000. Costa Rica: Imprenta Nacional.

Santos, M. (1993). Hacer visible lo cotidiano. Teoría y práctica de la evaluación cualitativa de los centros escolares. Madrid: Akal Ediciones. 